\title{
EXFOLIATIVE ERYTHRODERMA PRECEDING DERMATOMYOSITIS: CASE REPORT
}

Viviane Alves Costa ${ }^{1, \star}$, Luciana Akita ${ }^{1}$, Gizelle Gouvea Rezende ${ }^{1}$, Viviane Queiroz de Oliveira Maia ${ }^{1}$, Danielly Dantas Pimentel ${ }^{1}$, lane Tamara Dondé ${ }^{1}$, Gustavo Roberto Lourenço ${ }^{1}$, Juliana de Jesus Boscolo ${ }^{1}$, Taísa Morete da Silva ${ }^{1}$, Roberto Acayaba de Toledo ${ }^{1}$

1.Faculdade de Medicina de São José do Rio Preto, São José do Rio Preto (SP), Brazil.

*Corresponding author: vivicosta_med@hotmail.com

\section{BACKGROUND}

Inflammatory myopathies are a group of rare autoimmune diseases characterized by proximal muscle weakness that can be associated with cutaneous manifestations and involvement of other organs. Dermatomyositis presents characteristic cutaneous findings that are highly suggestive of the disease. Cutaneous involvement may precede or be simultaneous with muscle involvement. The aim of this study was to present the case of a patient with chronic cutaneous involvement who has developed musculoskeletal manifestations.

\section{CASE REPORT}

A 58-year-old white woman with a history of diabetes mellitus was referred by the dermatology service for presenting generalized exfoliative dermatitis with xerosis, especially in photoexposed areas for 2 years, with no further conclusions about her clinical presentation at the time. One month ago, she developed intermittent low fever in the absence of infectious conditions, arthralgia, myalgia and proximal muscle weakness. She was good breathing by herself. Xerotic skin, mild desquamation and hyperchromic spots all over her body, more evident in photoexposed areas (Fig. 1). Hypochromic areas on the eyelids, nose and jaw (Fig. 2). Grade 4 muscle strength on pectoral and pelvic girdle. Laboratory tests revealed mild normocytic normochromic anemia; slightly elevated concentration of muscle enzymes; negative hepatitis and HIV serological tests; normal thyroid function. Table 1 shows the whole autoantibody testing. The histopathological report revealed characteristic dermatomyositis findings. Chest computed tomography (CT) was normal. Screening for stomach, breast, uterus and bowel neoplasms was negative. Primary Sjögren's syndrome was discarded. Due to the elevated risk of bronchoaspiration, pulse therapy with methylprednisolone was performed, adding methotrexate to aid in weaning from corticosteroid.

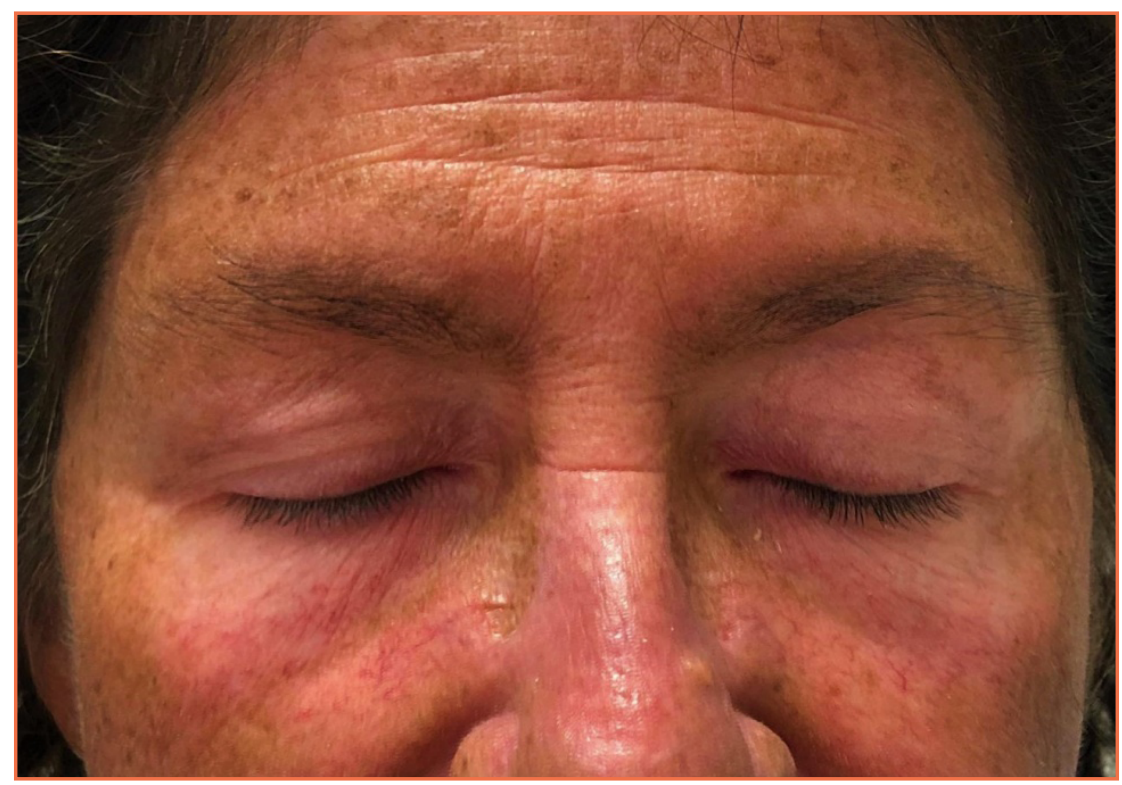

Figure 1. Hypochromic areas on the eyelids, nose and jaw. 


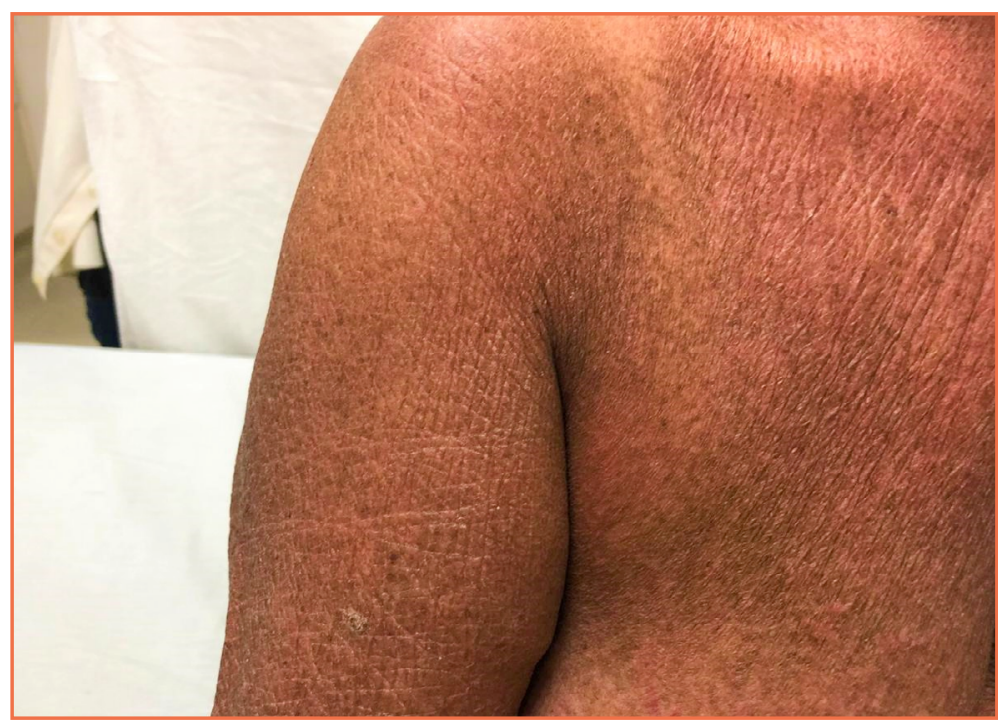

Figure 2. Mild desquamation and hyperchromic spots on the chest and forearm.

Table 1. Antibody panel.

\begin{tabular}{cc}
\hline ANA: $1 / 1280$ nucleolar pattern & \\
\hline Anti-Jo-1: negative & Anti-Mi-2: negative \\
\hline Anti-Ro: $>240$ & Anti-La: negative \\
\hline Anti-Scl: negative & Anticentromere: negative \\
\hline C3 $120 / /$ C4 16 & Anti-RNP: negative \\
\hline Anti-Sm: negative & Anti-DNA: negative \\
\hline Rheumatoid factor: nonreagent &
\end{tabular}

\section{CONCLUSION}

The case presented is an example of dermatomyositis with a rare cutaneous involvement that happened 2 years before the onset of muscle symptoms. Retrospectively, the facial lesions were considered to be the heliotrope rash and malar rash seen in dermatomyositis. One month after pulse therapy, the patient returned without dysphagia and with a great improvement of the skin lesions (Fig. 3), arthralgia, myalgia and muscle weaknesses.

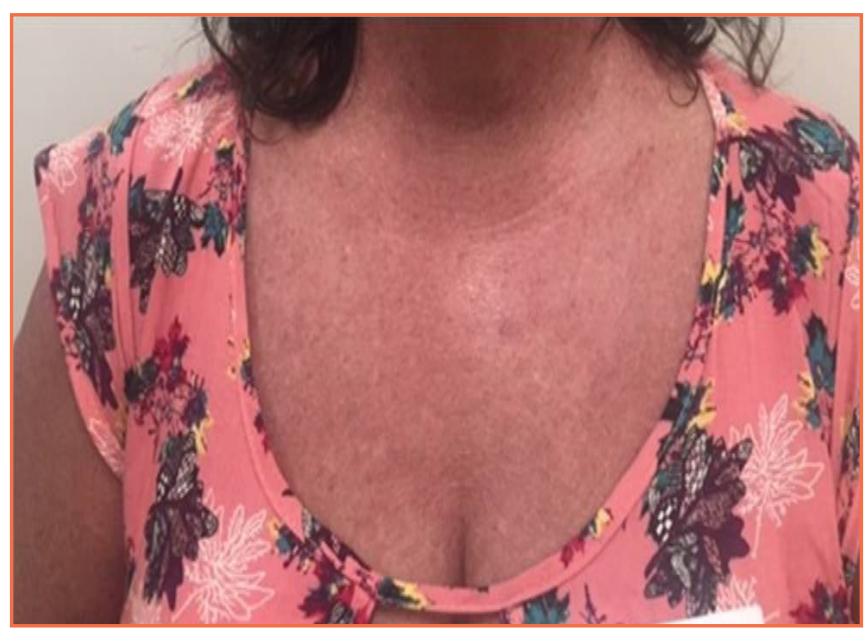

Figure 3. Chest region after pulse therapy. 